médecine/sciences $1995: 11: 1056$

\section{COURRIER}
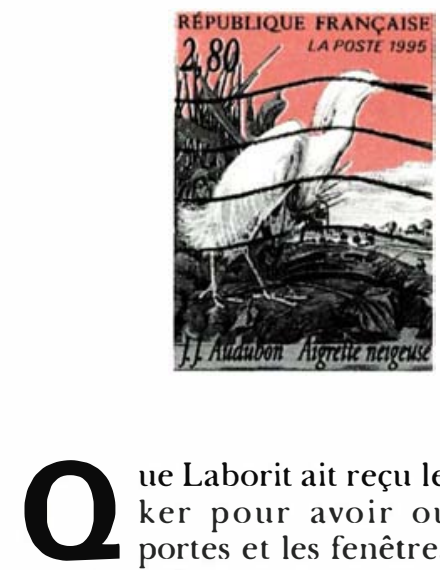

ue Laborit ait reçu le prix Lasker pour avoir ouvert les portes et les fenêtres des hospices psychiatriques, cela fut bien et cela fut certainement mieux que le goulag qui s'ensuivit naguère.

Que Laborit fut glorifié par un film racontant la rencontre d'un Rat et des Hommes, je dirais volontiers, comme une paraphrase du livre "Des souris et des hommes », que ce film n'a pas donné la place qu'il fallait à l'Idée

C'est cette Idée que je veux commenter pour honorer la mémoire d'Henri Laborit.

Je l'ai rencontré parce que j'avais lu qu'il préconisait, pour bloquer la réaction au stress de l'axe hypophysothalamo-surrénalien, d'associer un morphinique du type Dolosal, un antihistaminique et de l'Atropine.

\title{
Henri Laborit nous a quittés
}

C'était déjà la neuroplégie qui donna naissance à l'hibernation artificielle et à la neuroleptanalgésie.

Le reste est venu après que Madame Courvoisier (Directrice des recherches pharmacologiques de Rhône-Poulenc) eut retenu le $4560 \mathrm{RP}$, connu ensuite sous le nom de Largactil.

Et nous étions comme des disciples mais aussi plein de critiques : $\mathrm{Hu}$ guenard (Anesthésiste en Neurochirurgie à la Pitié puis Prof esseur et Directeur du SAMU - Henri Mondor) pour codifier ces formes nouvelles d'anesthésie à Paris, Campan (Anesthésiste en Neurochirurgie - hôpital Purpan - Toulouse), Ducailar (Anesthésiste - hôpital Lapeyronnie Montpellier), Deleuze (Anesthésiste à Cochin puis à la Pitié en Neurochirurgie), et sur le plan expérimental, Georges (Pharmacologue - Centre de Thérapeutique expérimentale à la Pitié - CTE) et moi-même avec Herold (Pharmacologue au CTE à la Pitié - la " miss " comme l'appelait Laborit), et bien d'autres qui me pardonneront de ne pas les avoir nommés.
Ainsi faut-il reconnaître, après bien des années qu'un produit pharmaceutique n'est qu'un instrument servile s'il n'est pas précédé par un concept, soyons modeste pour Henri qui ne l'a pas toujours été, par une Idée

"Un jour sur le pont d'un galion de l'Armada espagnole, un poisson volant est tombé.

Un matelot l'a donné à son officier qui le montra à son capitaine, un spécialiste, qui consulta ses livres. Passant par la voie hiérarchique, il dit à l'officier-Monsieur, donnez i ce matelot, pour qu'il le rejette à la mer, ce poisson qui n'existe pas.

Citation anonyme

"La dermière chose qu'on trouve en faisant un ouvrage est de savoir celle qu'il faut mettre la première. "

Pascal (Pensées - 19)

\section{Prof esseur Jean Cahn}

Neurobiologisle, 11, me Pérignon, 75015 Paris, France. 